PROCEEDINGS OF THE

AMERICAN MATHEMATICAL SOCIETY

Volume 128, Number 3, Pages 709-712

$\mathrm{S}$ 0002-9939(99)05098-4

Article electronically published on July 28, 1999

\title{
PRODUCT-TRACE-RINGS AND A QUESTION OF G. S. GARFINKEL
}

\author{
RALF KEMPER
}

(Communicated by Ken Goodearl)

Dedicated to H. Röhrl on the occasion of his 70th birthday

\begin{abstract}
It is an open question as to whether every left coherent ring $R$ satisfying the intersection property for finitely generated left ideals of $R$ is a right-product-trace-ring or not. $R$ is a right-product-trace-ring iff every product of trace-right- $R$-modules ( = universally torsionless-right- $R$-modules) is a trace-right- $R$-module. This question is shown to have a negative answer. Furthermore, looking at all valuation domains, the complete product-tracerings, the product-trace-rings and the product-content-rings are characterized.
\end{abstract}

\section{INTRODUCTION}

In this paper all rings have an identity and all modules are unitary. A right- $R$ module $M$ over an arbitrary $\operatorname{ring} R$ is called universally torsionless $(U T L)$ if the natural mapping $M \otimes A \rightarrow \operatorname{Hom}\left(M^{*}, A\right)$, where $M^{*}:=\operatorname{Hom}(M, R)$, is monic for all left- $R$-modules $A$ ([4], p. 119). Bass calls $M$ torsionless if the above mapping is monic for $A:=R$ and reflexive if the same mapping is an isomorphism. $M$ is a $U T L$-right- $R$-module if and only if $M$ is a trace-right- $R$-module, i.e. $x \in M \cdot M^{*}(x)$, where $M^{*}(x):=\left\{f(x) \mid f \in M^{*}\right\}$, for every $x \in M$ ([4], Th. 3.2). Various equivalent conditions for $M$ to be a trace-right- $R$-module can be found in [4], Th. 3.2. Tracemodules over commutative rings were also investigated in [8] and [6]. In [6] a trace-module is called a flat strict Mittag-Leffler module.

A direct sum of right-modules is a trace-right-module, if and only if each summand is ([4], 5.1). The situation for products is much more complicated. $R$ is a right-product-trace-ring (i.e. every product of trace-right- $R$-modules is a traceright- $R$-module) if it is left Noetherian ([4], Cor., p. 133). If $R$ is a right-producttrace-ring, then $R$ is left coherent (i.e. each finitely generated (f.g.) left ideal of $R$ is finitely presented) and satisfies the intersection property for f.g. left ideals (i.e. every intersection of f.g. left ideals is f.g.) ([4], Th. 5.13). G. S. Garfinkel asked in his paper ([4], p. 137) whether the converse of [4], Th. 5.13, is true or not. It is the aim of this paper to answer this question in the negative. Furthermore, looking

Received by the editors November 25, 1997 and, in revised form, May 1, 1998.

1991 Mathematics Subject Classification. Primary 12J25, 13A18, 13C13, 13E05, 13F30, 13J10, $46 \mathrm{~N} 05$.

Key words and phrases. Trace-module, universally torsionless module, product-trace-ring, ((maximally) complete) valuation domain, spherically complete field, content-module, contentideal. 
at all valuation domains, the product-trace-rings, complete product-trace-rings and product-content-rings are characterized.

$\mathbb{R}\left(\mathbb{Z}\right.$ resp.) is the set of real numbers (integers resp.) and we put $\mathbb{R}^{+}:=\{x \in$ $\mathbb{R} \mid x \geq 0\}$ and $\mathbb{Z}^{+}:=\mathbb{Z} \cap \mathbb{R}^{+}$. A valuation ring $R$ is a commutative ring with 1 whose ideals are totally ordered by inclusion. If, in addition, $R$ is an integral domain, it is called a valuation domain. Every valuation domain $R$ is the domain $R_{v}:=\{x \in K \mid v(x) \geq 0\}$ of a surjective valuation $v: K \rightarrow \Gamma \cup\{\infty\}$ ([3], I, Prop. 3.1 ). Here, $K$ is the quotient field of $R, \Gamma$ is a totally ordered (additively written) abelian group, $\infty$ is a symbol regarded as larger than any element of $\Gamma$ and $v$ fulfills the well-known axioms. In the following $R$ always denotes a valuation domain of a surjective valuation $v: K \rightarrow \Gamma \cup\{\infty\}$. $R$ is called maximally complete if it does not admit any proper immediate extension ([3], I, p. 5). This is the case if and only if $R$ is pure-injective (= algebraically compact) as an $R$-module, and this is equivalent to $K$ being spherically complete (see [3] and [7]). For all standard definitions the reader is referred to [2], [3], [7] and [9].

In case $\operatorname{rank}(v)=1, \prod_{i \in I} K:=\left\{\left(z_{i}\right)_{i \in I} \mid z_{i} \in K(i \in I)\right.$ and $\inf \left\{v\left(z_{i}\right) \mid i \in I\right\}>$ $-\infty\}$ is the product in the category of $K$-Banach spaces for every set $I$; here the norm of $\left(z_{i}\right)_{i \in I} \in \prod_{i \in I} K$ is $\inf \left\{v\left(z_{i}\right) \mid i \in I\right\}$ ([9], p. 52). The existence of surjective valuations $v: K \rightarrow \mathbb{R} \cup\{\infty\}$ such that $R$ is complete, but not maximally complete (or, equivalently, $K$ is complete, but not spherically complete), is well-known ([9], Th. 1.3 and p. 11, Rem. (ii) and p. 25, Ex. 2. G). Hence the following proposition answers the question of G. S. Garfinkel in the negative.

Proposition 1. Let $v: K \rightarrow \mathbb{R} \cup\{\infty\}$ be an arbitrary surjective valuation, such that $R$ is complete, but not maximally complete. Then $R$ is coherent and satisfies the intersection property for finitely generated ideals of $R$, but $R$ is not a producttrace-ring.

Proof. Since the value group $\mathbb{R}$ is complete as a lattice, according to [4], Ex. 5.3, we only have to show that $R$ is not a product-trace-ring. Assume that $R^{\mathbb{N}}$ is a trace- $R$ module. Then, for some (every resp.) $\beta:=\left(\beta_{n}\right)_{n \in \mathbb{N}} \in R^{\mathbb{N}}$ with $v\left(\beta_{n}\right)>v\left(\beta_{n+1}\right)$ for every $n \in \mathbb{N}$, there exist $\gamma:=\left(\gamma_{n}\right)_{n \in \mathbb{N}} \in R^{\mathbb{N}}$ and $f \in \operatorname{Hom}\left(R^{\mathbb{N}}, R\right)$ with $\beta=f(\beta) \gamma$. $\left(B, u_{B}\right)$ with $B:=\prod_{n \in \mathbb{N}} K$ and $u_{B}\left(\left(z_{n}\right)_{n \in \mathbb{N}}\right):=\inf \left\{v\left(z_{n}\right) \mid n \in \mathbb{N}\right\}$ is a $K$-Banach space. Define a mapping $g: B \rightarrow K$ as follows: For $x:=\left(x_{n}\right)_{n \in \mathbb{N}} \in R^{\mathbb{N}}$ put $g(x):=$ $f(x)$. For $x \in B \backslash R^{\mathbb{N}}$ there is a $\tau \in K \backslash\{0\}$ with $v(\tau)=\inf \left\{v\left(x_{n}\right) \mid n \in \mathbb{N}\right\}<0$. Then one gets $\frac{1}{\tau} x=\left(\frac{1}{\tau} x_{n}\right)_{n \in \mathbb{N}} \in R^{\mathbb{N}}$. In this case define $g(x):=\tau f\left(\frac{1}{\tau} x\right)$. For every $\sigma \in K \backslash\{0\}$ with $v(\sigma) \leq v(\tau)$ one has $\frac{1}{\sigma} x \in R^{\mathbb{N}}$, and from $\sigma f\left(\frac{1}{\sigma} x\right)=\tau f\left(\frac{1}{\tau} x\right)$ one easily sees that $g: B \rightarrow K$ is well-defined and $K$-linear. Take an arbitrary $x=\left(x_{i}\right)_{i \in \mathbb{N}} \in R^{\mathbb{N}}$ and assume the existence of $\lambda \in R$ with $v(f(x))<v(\lambda) \leq$ $\inf \left\{v\left(x_{i}\right) \mid i \in \mathbb{N}\right\}$. This leads to the contradiction $v(f(x))=v\left(\lambda f\left(\left(\frac{x_{i}}{\lambda}\right)_{i \in \mathbb{N}}\right)\right) \geq v(\lambda)$. Thus $v(f(x)) \geq \inf \left\{v\left(x_{i}\right) \mid i \in \mathbb{N}\right\}$ follows for every $x=\left(x_{i}\right)_{i \in \mathbb{N}} \in R^{\mathbb{N}}$. Therefore, for each $x:=\left(x_{n}\right)_{n \in \mathbb{N}} \in B \backslash R^{\mathbb{N}}$ with $u_{B}(x)=v(\tau)$

$$
\begin{aligned}
v(g(x))=v\left(\tau f\left(\frac{1}{\tau} x\right)\right)=v(\tau)+v\left(f\left(\frac{1}{\tau} x\right)\right) & \geq v(\tau)+\inf \left\{v\left(\frac{1}{\tau} x_{n}\right) \mid n \in \mathbb{N}\right\} \\
& =\inf \left\{v\left(x_{n}\right) \mid n \in \mathbb{N}\right\}=u_{B}(x)
\end{aligned}
$$

holds. $v(g(x)) \geq u_{B}(x)$ is also valid for $x \in R^{\mathbb{N}}$. Hence $g: B \rightarrow K$ is a contraction. Since $K$ is not spherically complete, there exist endomorphisms $g_{n}: K \rightarrow K$ of the $K$-Banach space $K, n \in \mathbb{N}$, with $\left(g_{n}\right)_{n \in \mathbb{N}} \in \bigoplus_{n \in \mathbb{N}} K^{\prime}$, and for every $z:=\left(z_{n}\right)_{n \in \mathbb{N}} \in$ $B, g(z)=\sum_{j \in \mathbb{N}} g_{j}\left(z_{j}\right)$ holds because of [9], Th. 4.22(i). This implies $\beta=g(\beta) \gamma=$ 
$\left(\sum_{j \in \mathbb{N}} g_{j}\left(\beta_{j}\right) \gamma_{n}\right)_{n \in \mathbb{N}}$. Obviously, there exists a $j_{0} \in \mathbb{N}$ with $v\left(\sum_{j \in \mathbb{N}} g_{j}\left(\beta_{j}\right)\right) \geq$ $g_{j_{0}}\left(\beta_{j_{0}}\right)$, and $g_{j_{0}}: K \rightarrow K$ is a contraction.

$$
v\left(\beta_{n}\right)=v\left(\sum_{j \in \mathbb{N}} g_{j}\left(\beta_{j}\right) \gamma_{n}\right) \geq v\left(\sum_{j \in \mathbb{N}} g_{j}\left(\beta_{j}\right)\right) \geq v\left(g_{j_{0}}\left(\beta_{j_{0}}\right)\right) \geq v\left(\beta_{j_{0}}\right)
$$

follows for every $n \in \mathbb{N}$. This contradicts the choice of $\beta \in R^{\mathbb{N}}$, i.e. $R$ is not a product-trace-ring.

Every field $K$ is Noetherian, thus a product-trace-ring ([4], Cor., p. 133). Hence, in the following we may restrict our attention to valuation domains $R \neq K$, i.e. the valuation $v: K \rightarrow \Gamma \cup\{\infty\}$ is not trivial.

Theorem 2. For every valuation domain $R \neq K$, the following are equivalent:

(i) $R$ is a complete product-trace-ring.

(ii) $R$ is coherent and satisfies the intersection property for finitely generated ideals of $R$, and the following condition a) or b) is fulfilled:

a) $R$ is complete and $v$ is discrete with rank $(v)=1$.

b) $v(R \backslash\{0\})=\mathbb{R}^{+}$and $R$ is maximally complete.

(iii) $(R$ is complete and $v$ is discrete with $\operatorname{rank}(v)=1)$ or $\left(v(R \backslash\{0\})=\mathbb{R}^{+}\right.$and $R$ is maximally complete).

(iv) $R$ is maximally complete and $v\left(R \backslash\{0\}\right.$ ) is (isomorphic to) $\mathbb{Z}^{+}$or $\mathbb{R}^{+}$.

Proof. (i) $\Rightarrow$ (ii): By [4], Th. 5.13, the product-trace-ring $R$ is coherent and satisfies the intersection property for f.g. ideals of $R$. Assume $\operatorname{rank}(v)>1$. Then there exist $\alpha, \beta \in R \backslash\{0\}$ with $0<v\left(\alpha^{n}\right)<v(\beta)$ for each $n \in \mathbb{N}$ ([5], Ex. 15, p. 203). Because of [4], Ex. 5.3, $\Gamma$ is complete as a lattice. Thus $s:=\sup \left\{v\left(\alpha^{n}\right) \mid n \in \mathbb{N}\right\}$ exists in $\Gamma$. From $s=\sup \left\{v\left(\alpha^{n+1}\right) \mid n \in \mathbb{N}\right\}=s+v(\alpha)$ one gets the contradiction $v(\alpha)=0$. Hence $\operatorname{rank}(v)=1$, and the remaining part of (ii) is a consequence of the above intersection property (resp. of the preceding proposition).

(ii) $\Rightarrow$ (iii) is trivial.

(iii) $\Rightarrow$ (i): If $\operatorname{rank}(v)=1, R$ is complete and $v$ is discrete, $R$ is a complete producttrace-ring by [4], Cor., p. 133. But the following proof in the case $v(R \backslash\{0\})=\mathbb{R}^{+}$ and $R$ maximally complete is also valid in the first case. Let $I$ be an arbitrary set and $0 \neq x:=\left(x_{i}\right)_{i \in I} \in R^{I}$. There exists a $\gamma \in R \backslash\{0\}$ with $\inf \left\{v\left(x_{i}\right) \mid i \in I\right\}=v(\gamma)$. Since $K$ is spherically complete, there is a contraction $g: \prod_{i \in I} K \rightarrow K$ with $g(x)=\gamma\left([1]\right.$, Cor. 2). The restriction $f: R^{I} \rightarrow R$ of $g$ is a (well-defined) morphism of $R$-modules. From $\left(\frac{x_{i}}{\gamma}\right)_{i \in I} \in R^{I}$ and $x=f(x)\left(\frac{x_{i}}{\gamma}\right)_{i \in I}$ one concludes that $R^{I}$ is a trace- $R$-module. Therefore, $R$ is a product-trace-ring ([4], Th. 5.4) and $R$ is obviously complete.

(iii) $\Leftrightarrow($ iv) is a consequence of [9], 2.4.

The conditions in (ii) ((iii), (iv) resp.) of the theorem above do not characterize all valuation domains which are product-trace-rings. Indeed, every non-complete valuation domain $R$ with $v(R \backslash\{0\})=\mathbb{Z}^{+}$is Noetherian, and therefore, by [4], Cor., p. 133, a product-trace-ring. But we are able to prove the following

Theorem 3. For every valuation domain $R \neq K$ the following are equivalent:

(i) $R$ is a product-trace ring.

(ii) $v(R \backslash\{0\})=\mathbb{Z}^{+}$or $\left(v(R \backslash\{0\})=\mathbb{R}^{+}\right.$and $R$ is maximally complete $)$. 
Proof. (ii) $\Rightarrow$ (i): If $v(R \backslash\{0\})=\mathbb{Z}^{+}, R$ is Noetherian, hence a product-trace-ring

([4], Cor., p. 133); otherwise, because of Theorem $2, R$ is a product-trace-ring.

(i) $\Rightarrow$ (ii): The proof of Theorem 2 , (i) $\Rightarrow\left(\right.$ ii), shows that $v(R \backslash\{0\})=\mathbb{Z}^{+}$or $v(R \backslash\{0\})=\mathbb{R}^{+}$holds. We may assume $v(R \backslash\{0\})=\mathbb{R}^{+}$. If $R$ is complete, $R$ is maximally complete by Proposition 1 . Assume that $R$ fails to be complete, and let $\left(x_{n}\right)_{n \in \mathbb{N}}$ be a sequence in $R$, such that $\left(v\left(x_{n}\right)\right)_{n \in \mathbb{N}}$ is strictly decreasing. Be-

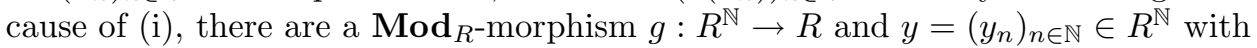
$x=g(x) y$. Since $K$ is obviously countably generated and $R$ is not complete, the $R$-module $R$ is slender ([3], XIV, Cor. 7.9), i.e., there is an $m \in \mathbb{N}$ with $g\left(e_{n}\right)=0$ for $n>m$. Put $z:=x-\sum_{i=1}^{m} x_{i} e_{i}$ and let $h: \prod_{i=m+1}^{\infty} R \rightarrow R$ be the restriction of $g: R^{\mathbb{N}} \rightarrow R$. Since $R$ is slender and because $h\left(R e_{i}\right)=R g\left(e_{i}\right)=0$ for $i>m$, one obtains $h=0$ ([3], XIV, Th. 7.4(ii)). Hence $x=g(x) y=g\left(z+\sum_{i=1}^{m} x_{i} e_{i}\right) y=$ $\left(h(z)+\sum_{i=1}^{m} x_{i} g\left(e_{i}\right)\right) y=\sum_{i=1}^{m} x_{i} g\left(e_{i}\right) y$ follows, which implies the contradiction $v\left(x_{n}\right)=v\left(\sum_{i=1}^{m} x_{i} g\left(e_{i}\right) y_{n}\right) \geq v\left(x_{i_{0}} g\left(e_{i_{0}}\right) y_{n}\right) \geq v\left(x_{i_{0}}\right)$ for some $i_{0} \in \mathbb{N}_{m}$ and every $n \in \mathbb{N}$. This finishes the proof.

For each right- $R$-module $M c(x):=\bigcap\{I \mid I$ is a left ideal of $R$ with $x \in M I\}$ is the content-ideal of $x \in M$ ([4], p. 137). $M$ is called a content-right- $R$-module if $x \in M c(x)$ for every $x \in M$. We call $R$ a right-product-content-ring if each product of content-right- $R$-modules is a content-right- $R$-module. A right- $R$-module $M$ is a trace-right- $R$-module if and only if $M$ is a content-right- $R$-module with $c(x)=\left\{f(x) \mid f \in M^{*}\right\}$ for every $x \in M$ ([4], 5.14 (4); [8], Rem. p. 66). For example, in case $\operatorname{rank}(v)=1$ and $v$ is discrete, an $R$-module $M$ is a content- $R$ module if and only if $M$ is division free (i.e. the only divisible element of $M$ is 0 ) ([8], Prop. 2.1). The following theorem is a simple consequence of [4], Ex. 5.3, Th. 5.15 , and of the first part of the proof of "(i) $\Rightarrow$ (ii)" in Theorem 2.

Theorem 4. For any valuation domain $R \neq K$ the following are equivalent:

(i) $R$ is a product-content-ring.

(ii) $\operatorname{rank}(v)=1$ and $\left(v\right.$ is discrete or $\left.v(R \backslash\{0\})=\mathbb{R}^{+}\right)$.

\section{REFERENCES}

[1] R.L. Ellis: The state of a bounded linear operator on a non-Archimedean normed space; J. Reine Angew. Math., 229 (1968), 155-162. MR 36:6958

[2] O. Endler: Valuation Theory; Springer-Verlag, Berlin, Heidelberg, New York (1972). MR 50:9847

[3] L. Fuchs, L. Salce: Modules over Valuation Domains; Lecture notes in pure and applied mathematics, 97, Marcel Dekker, New York (1985). MR 86h:13008

[4] G. S. Garfinkel: Universally Torsionless and Trace Modules; Trans. Amer. Math. Soc., 215 (1976), 119-144. MR 53:8136

[5] R. Gilmer: Multiplicative Ideal Theory; Pure and Applied Mathematics, 12, Marcel Dekker, New York (1972). MR 55:323

[6] L. Gruson, M. Raynaud: Critères de platitude et de projectivité. Techniques de "platification" d'un module; Invent. Math., 13 (1971), 1-89. MR 46:7219

[7] L. Narici, E. Beckenstein, G. Bachmann: Functional Analysis and Valuation Theory; Pure and Applied Mathematics, 5, Marcel Dekker, New York (1971). MR 50:14142

[8] J. Ohm, D.E. Rush: Content Modules and Algebras; Math. Scand., 31 (1972), 49-68. MR 49:9028

[9] A.C.M. van Rooij: Non-Archimedean Functional Analysis; Pure and Applied Mathematics, 51, Marcel Dekker, New York (1978). MR 81a:46084

Fernuniversität, Fachbereich Mathematik, D 58084 Hagen, Germany 